\title{
THE
}

\section{Dynamical ansatz for path integrals and nonperturbative trace formulas}

Yuri A. Dabaghian

University of Rhode Island

Follow this and additional works at: https://digitalcommons.uri.edu/phys_facpubs

Terms of Use

All rights reserved under copyright.

\section{Citation/Publisher Attribution}

Dabaghian, Y. A. (1999). Dynamical ansatz for path integrals and nonperturbative trace formulas. Phys. Rev. E, 60(!), 324-334. doi: 10.1103/PhysRevE.60.324

Available at: https://doi.org/10.1103/PhysRevE.60.324

This Article is brought to you for free and open access by the Physics at DigitalCommons@URI. It has been accepted for inclusion in Physics Faculty Publications by an authorized administrator of DigitalCommons@URI. For more information, please contact digitalcommons-group@uri.edu. 


\title{
Dynamical ansatz for path integrals and nonperturbative trace formulas
}

\author{
Yu. A. Dabaghian \\ Department of Physics, University of Rhode Island, Kingston, Rhode Island 02881
}

(Received 17 December 1998)

\begin{abstract}
It is shown that a recently discovered representation of the Green's function is equivalent to a certain "dynamical ansatz" for the corresponding path integral, which brings about a convenient method of nonperturbative approximations. Based on this observation, a set of nonperturbative approximations to the trace of the Green's function is established. [S1063-651X(99)06805-1]
\end{abstract}

PACS number(s): 05.45.Mt, 11.15.Tk, 03.65.Db

\section{INTRODUCTION}

In order to determine the energy spectrum of a quantum system one considers the trace of its Green's function

$$
G(E)=\int G(x, x \mid E) d x
$$

which, in terms of a Feynman path integral formalism, is given by a sum over all the closed paths in the configurational space. In the semiclassical limit $\hbar \rightarrow 0$, this sum reduces to the one over all the closed classical trajectories $[5-7]$ :

$$
G_{q c l}(E)=\sum_{\text {per.orbits }} \frac{e^{i S_{c l}+i \nu}}{\sqrt{\operatorname{det}\left(1-\mathbf{M}_{p}\right)}} .
$$

Here $S_{c l}$ is the classical action functional evaluated on an orbit $\gamma$ and $\mathbf{M}_{p}$ is the stability matrix of that orbit.

Inasmuch as the basic tool of the analysis of the semiclassical behavior, the semiclassical trace $G_{q c l}(E)$, is built from a set of classical objects, one would expect to be able to trace the correspondence of certain quantum properties to the dynamical features of the corresponding classical system.

However, this correspondence and the above formula are valid only in the limit $\hbar \rightarrow 0$. An interesting question is whether it is possible to go beyond this limit and to obtain some $\hbar \neq 0$ approximations to the exact quantum trace without using perturbative techniques; whether it is possible to extend the sum from the set of classical orbits to some larger set of paths to produce a consistent approximation to the exact trace outside the limit $\hbar \rightarrow 0$.

\section{THE GREEN'S FUNCTION}

The starting point of the following discussion is a representation of the causal Green's function, obtained recently in publications [1], and presented here using the example of Dirac's equation. The derivation of this representation in [1] was based on the Schwinger-Fradkin representation of Green's function $[2,3]$. For a particle of a mass $m$ and charge $g$ moving in the external field with vector potential $A_{\mu}$ such a Green's function can be written in terms of a functional integral over the velocity $v_{\mu}(s)$ of the particle as

$$
\begin{aligned}
G_{A}(x, y)= & i \int_{0}^{\infty} d s e^{-i s m^{2}} N \int D v e^{(i / 4) \int_{0}^{s} v_{\mu}^{2}\left(s^{\prime}\right) d s^{\prime}} \\
& \times\left[m-i \gamma_{\mu}\left(p_{\mu}-\frac{e}{c} A_{\mu}\right)\right] \\
& \times e^{-i g \int_{0}^{s} d s^{\prime} v_{\mu}\left(s^{\prime}\right) A_{\mu}\left(y-\int_{0}^{s^{\prime}} v\right)} \\
& \times \delta\left(x-y+\int_{0}^{s^{\prime}} v\right) .
\end{aligned}
$$

Here $N$ is the normalizing coefficient $N^{-1}$ $=\int D v e^{(i / 4) \int_{0}^{s} v_{\mu}^{2}\left(s^{\prime}\right) d s^{\prime}}$. One can use the $\delta\left(x-y+\int_{0}^{s^{\prime}} v\right)$ to write the previous formula in terms of the path integral:

$$
\begin{aligned}
G_{A}(x, y)= & i \int_{0}^{\infty} d s e^{-i s m^{2}} N \int D X_{\mu} e^{(i / 4) \int_{\gamma} \dot{X}_{\mu}^{2} d s^{\prime}} \\
& \times\left[m-i \gamma_{\mu}\left(p_{\mu}-\frac{e}{c} A_{\mu}\right)\right] e^{-i g \int_{0}^{s} d X_{\mu} A_{\mu}(X)},
\end{aligned}
$$

where a path $\gamma$ connects the initial $X_{\mu}(0)$ and the final $X_{\mu}(s)$ points of the evolution.

The representation in terms of a functional path integral is given by

$$
\begin{aligned}
G_{c}\left(p, p^{\prime}\right)= & i \int_{0}^{\infty} d s e^{-i m^{2} s} \int D X_{\mu} \\
& \times \int D \xi_{\mu} e^{-i \int_{\gamma}\left(\dot{X}_{\mu} / 4+\xi_{\mu}\right) d X_{\mu}} e^{i q_{\mu} / 2\left(X_{\mu}(s)+X_{\mu}(0)\right)} \\
& \times\left(e^{-g \int \sigma F(X)}\right)_{+} \delta\left(X_{\mu}-f_{\mu}^{s}\left(X, A, \xi_{\mu}\right)\right) \\
& \times\left\{m-i \gamma_{\mu}\left(\frac{1}{2} \dot{X}_{\mu}+\frac{q_{\mu}}{2}+\xi_{\mu}\right)\right\} .
\end{aligned}
$$

Here $\xi_{\mu}=\xi_{\mu}(s)$ is a certain auxiliary function, and the argument of the $\delta$ functional is defined by the formal dynamical flow generated by the dynamical system,

$$
\frac{d X_{\mu}}{d s}=p_{\mu}+p_{\mu}^{\prime}-2 \xi_{\mu}(s)-2 g A_{\mu}(X),
$$

the time integral of these equations, 


$$
\begin{aligned}
X_{\mu}(s) & =X_{\mu}(0)+\left(p_{\mu}+p_{\mu}^{\prime}\right) s-2 \int_{0}^{s} \xi_{\mu} d s^{\prime}-2 g \int_{0}^{s} A_{\mu}(X) d s^{\prime} \\
& \equiv f_{\mu}^{t}(X, A, \xi) .
\end{aligned}
$$

The connection between the representation (2) and the standard one can be easily established. Using the fact that the argument of the $\delta$ functional,

$$
\delta\left(X_{\mu}(s)-f_{\mu}^{t}(X, A, \xi)\right)
$$

is linear in $\xi_{\mu}$, one can immediately perform the functional integration over $\xi_{\mu}$. The determinant $\operatorname{det}\left[\delta\left(x_{\mu}\right.\right.$ $\left.\left.-f_{\mu}(x, \xi, A)\right) / \delta \xi_{\nu}\right]=1$, and hence, inserting

$$
\xi_{\mu}=\frac{p_{\mu}+p_{\mu}^{\prime}}{2}-g A_{\mu}(X)-\frac{1}{2} \dot{X}_{\mu}
$$

into the phase $\int_{\gamma}\left(\dot{X}_{\mu} / 4+\xi_{\mu}\right) d X_{\mu}$, one gets

$$
\begin{aligned}
S & =\int_{\gamma}\left(\frac{\dot{X}_{\mu}}{4}+\frac{p_{\mu}+p_{\mu}^{\prime}}{2}-g A_{\mu}(X)-\frac{1}{2} \dot{X}_{\mu}\right) d X_{\mu} \\
& =\int_{\gamma}\left(-\frac{\dot{X}_{\mu}}{4}+\frac{p_{\mu}+p_{\mu}^{\prime}}{2}-g A_{\mu}(X)\right) d X_{\mu} \\
& =-\int_{\gamma}\left(\frac{\dot{X}_{\mu}}{4}+g A_{\mu}(X)\right) d X_{\mu}+\frac{p_{\mu}+p_{\mu}^{\prime}}{2} \Delta X_{\mu} .
\end{aligned}
$$

Combining the last term $-i\left(p_{\mu}+p_{\mu}^{\prime} / 2\right) \Delta X_{\mu}$ with the similar phase $i q_{\mu}\left[X_{\mu}(s)+X_{\mu}(0) 2\right]$, one gets

$$
\begin{aligned}
& -\frac{p_{\mu}+p_{\mu}^{\prime}}{2}\left(X_{\mu}(s)-X_{\mu}(0)\right)+\frac{p_{\mu}-p_{\mu}^{\prime}}{2}\left(X_{\mu}(s)+X_{\mu}(0)\right) \\
& \quad=p_{\mu} X_{\mu}(0)-p_{\mu}^{\prime} X_{\mu}(s),
\end{aligned}
$$

which brings us to

$$
\begin{aligned}
G_{c}= & i \int_{0}^{\infty} d s \int D X_{\mu} e^{-i m^{2} s} \\
& \times e^{i \int_{\gamma}\left(\dot{X}_{\mu} / 4+g A_{\mu}(X)\right) d X_{\mu}} e^{-i\left(p_{\mu}^{\prime} X_{\mu}(s)-p_{\mu} X_{\mu}(0)\right)} \\
& \times\left\{m-i \gamma_{\mu}\left(p_{\mu}-g A_{\mu}(X)\right)\right\}\left(e^{-g \int \sigma F(X)}\right)_{+} .
\end{aligned}
$$

The exponential $e^{-i\left(p_{\mu}^{\prime} X_{\mu}(s)-p_{\mu} X_{\mu}(0)\right)}$ is essentially a reminder of the fact that Green's function is in the momentum representation, $G_{c}=G_{c}\left(p, p^{\prime}\right)$. In coordinate representation one would obviously have the standard expression (1) for $G_{c}(x, y)$ :

$$
\begin{aligned}
G_{c}= & i \int_{0}^{\infty} d s \int D X_{\mu} e^{-i m^{2} s} e^{i \int_{\gamma}\left(\dot{X}_{\mu} / 4+g A_{\mu}(X)\right) d X_{\mu}} \\
& \times\left\{m-i \gamma_{\mu}\left(p_{\mu}-g A_{\mu}(X)\right)\right\}\left(e^{-g \int \sigma F(X)}\right)_{+},
\end{aligned}
$$

where $\gamma(s)$ is a path connecting $x=X_{\mu}(0)$ and $y=X_{\mu}(s)$. As one can see, the equivalency of the representation to the standard one is quite straightforward.
The constraint (4) actually provides another possible way to represent the Green's function, since one can consider the phase of the exponential in Eq. (2) modulo this constraint. Proceeding as before, one gets, using Eq. (5);

$$
\begin{aligned}
G_{c}= & i \int_{0}^{\infty} d s \int D X_{\mu} \int D \xi_{\mu} e^{-i m^{2} s} e^{i \int_{\gamma}\left(\dot{X}_{\mu} / 4+g A_{\mu}(X)\right) d X_{\mu}} \\
& \times e^{-i\left(p_{\mu}^{\prime} X_{\mu}(s)-p_{\mu} X_{\mu}(0)\right)} \delta\left(X_{\mu}-f_{\mu}^{s}(X, A, \xi)\right) \\
& \times\left\{m-i \gamma_{\mu}\left(p_{\mu}-g A_{\mu}(X)\right)\right\}\left(e^{-g \int \sigma F(X)}\right)_{+}
\end{aligned}
$$

Although the latter expression still contains the $\delta$ functional and the integral over $\xi_{\mu}$ was not taken yet, it bears a very close resemblance to the standard representation (1), and, therefore, it will be convenient for various purposes to consider the representation ( $7 \mathrm{~b})$ along with the original:

$$
\begin{aligned}
G_{c}= & i \int_{0}^{\infty} d s \int D X_{\mu} \int D \xi_{\mu} \\
& \times e^{-i m^{2} s} e^{-i \int_{\gamma}\left(\dot{X}_{\mu} / 4+\xi_{\mu}\right) d X_{\mu} e^{\left(i q_{\mu} / 2\right)\left(X_{\mu}(s)+X_{\mu}(0)\right)}} \\
& \times\left(e^{-g \int \sigma F(X)}\right)_{+} \delta\left(X_{\mu}-f_{\mu}^{s}(X, A, a)\right) \\
& \times\left\{m-i \gamma_{\mu}\left(\frac{1}{2} \frac{d X_{\mu}}{d s}+\frac{q_{\mu}}{2}+\frac{d R_{\mu}}{d s}\right)\right\},
\end{aligned}
$$

The expression (7a) could be obtained from the original (1) by inserting a "unity decomposition,"

$$
1=\int D \xi_{\mu} \delta\left(X_{\mu}(s)-f_{\mu}^{t}(X, A, \xi)\right),
$$

into the integrand. A representation equivalent to Eq. (2) was rigorously derived in [1]. However, one can think of it as of a certain ansatz, or an analytical trick that allows one to bring to light certain remarkable properties and physically interpretable structures of the functional integral. Due to the $\delta\left(X_{\mu}(s)-f_{\mu}^{t}(X, A, \xi)\right)$ constraint, one enjoys a very special feature of the representation, namely, that every path $\gamma:\left\{X_{\mu}\left(s^{\prime}\right), 0 \leqslant s^{\prime} \leqslant s\right\}$ in Eq. (2) is a solution to a certain set of dynamical equations (3). This circumstance allows one to consider every path on the configurational space from a certain dynamical point of view. It should be emphasized here that although this statement might seem restrictive, every path in configurational space contributes to the integrals (2) and (6). Indeed, for every path $\gamma: X_{\mu}\left(s^{\prime}\right), 0 \leqslant s^{\prime} \leqslant T$, the expression

$$
\frac{d X_{\mu}\left(s^{\prime}\right)}{d s^{\prime}}+g A_{\mu}\left(X\left(s^{\prime}\right)\right) \equiv \frac{p_{\mu}+p_{\mu}^{\prime}}{2}-\xi_{\mu}\left(X\left(s^{\prime}\right)\right)
$$

defines a certain function $\xi_{\mu}\left(s^{\prime}\right)$ of $s^{\prime}$ on the interval 0 $\leqslant s^{\prime} \leqslant s$, which corresponds to that particular path.

Although Eq. (7a) is seemingly very similar to the standard representation, it in fact allows one to establish a new perspective on the question of evaluating the functional integral via an uncommon sequence of nonperturbative approximations. 


\section{APPROXIMATIONS}

If it turns out to be impossible to explicitly evaluate the functional integral (1), one starts to apply various approximation techniques. The only approach that the original functional integral (1) naturally suggests is to look for the possible perturbative expansions near the saddle point of the phase. The saddle point itself, which corresponds to the zeroth order of perturbative expansion, describes the semiclassical regime [4]. All the higher orders of the perturbation series allow one to consider the vicinity of the saddle point. Physically speaking, a perturbative expansion represents a certain way to introduce quantum fluctuations into the system.

On the other hand, when facing the need to apply a certain perturbation technique, one has to chose the approach that is appropriate in a given physical situation. It is the character of the dynamics (classical or quantum) of the system in question that justifies the means of approximation. The perturbation techniques that are based on approximating the action functional near the saddle point might not be appropriate in some cases. By applying "perturbative" methods where the whole interaction (or just a part of it, such as the higher-order nonlinear terms in the action) are considered as a perturbation, one has to assume some kind of regularity of the dynamics, which should allow one to consider sequentially different parts of interaction. However, in some cases, such as the ones of the chaotic systems, one cannot make this assumption. All of the terms are essential in determining the global dynamical characteristics of the system. By using the perturbative methods one can unwillingly average off or destroy the evidence of some effects.

There exist methods, such as Gutzwiller's trace formula, that allow one to consider the saddle point in all its complexity, but they do not extend beyond the $\hbar=0$ point-unless the same old perturbative techniques are used. It is difficult to go further than the semiclassical description of the Green's function if one would need is to use some nonperturbative techniques. As it turns out, the representation (7a) suggests a simple set of nonperturbative approximations, which are described below.

The Fourier series expansion of the auxiliary function $\xi_{\mu}(\tau)$

$$
\begin{gathered}
\xi_{\mu}(\tau)=\frac{1}{\sqrt{T}} \sum_{n=0}^{N}\left(P_{\mu, n} \cos \left(\omega_{n} \tau\right)+Q_{\mu, n} \sin \left(\omega_{n} \tau\right)\right), \\
\omega_{n}=\omega(n+1 / 2), \quad \omega=\frac{2 \pi}{T},
\end{gathered}
$$

defines a set of the coefficients $P_{\mu, n}$. The frequency set $\omega_{n}$ $=(2 \pi / T)(n+1 / 2)$ emerged in the regrows derivation [1]. In terms of the expansion (8), the functional integration over the $\xi_{\mu}$ in Eqs. (7a) and (7b) should be understood as an integration over all the expansion coefficients $P_{\mu, n}$ :

$$
\int D \xi_{\mu} \equiv \int \cdots \int \Pi_{n} \Pi_{\mu=1}^{4} \frac{d P_{\mu, n} d Q_{\mu, n}}{2 \pi} .
$$

As is easy to see from Eq. (7a), if one uses this measure of the functional integration over $\xi_{\mu}$, there exists a remark- able possibility of dropping the $P_{n}$ dependence in Eq. (7a) [and consequently in Eq. (7b)] for any $n$ 's, without changing the overall structure of these formulas. Such "structural invariance" is very convenient. For instance, it implies the possibility to approximate the function $\xi_{\mu}(\tau)$ by it's Fourier polynomial $\xi_{\mu}^{N}(\tau)$ of the degree $N$ by dropping all the coefficients $P_{n}$ in Eq. (7) for $|n|>N$ :

$$
\xi_{\mu}^{N}(\tau)=\frac{1}{\sqrt{T}} \sum_{n=0}^{N}\left(P_{\mu, n} \cos \left(\omega_{n} \tau\right)+Q_{\mu, n} \sin \left(\omega_{n} \tau\right)\right)
$$

Consequently, the exact phase of the integrand of Eq. (7a) would be replaced by a certain "Fourier-type" approximation,

$$
S \equiv \int_{\gamma}\left(\frac{\dot{X}_{\mu}}{4}+\xi_{\mu}(s)\right) d X_{\mu} \rightarrow S_{N} \equiv \int_{\gamma}\left(\frac{\dot{X}_{\mu}}{4}+\xi_{\mu}^{N}(s)\right) d X_{\mu},
$$

and thus one would obtain a nontrivial sequence of approximations to the Green's function. The index $n$ enumerating the variables $P_{n}$ is not a perturbative index, and so these approximations are nonperturbative. Omitting the variables $P_{n}$ in the argument of the $\delta$ constraint implies that the path integration in Eq. (7a) or in Eq. (7b) is restricted to the paths that are the solutions to the system,

$$
\begin{aligned}
\frac{d X_{\mu}}{d \tau}+g A_{\mu}(X)= & \frac{p_{\mu}+p_{\mu}^{\prime}}{2}+\frac{1}{\sqrt{T}} \sum_{n=0}^{N}\left(P_{\mu, n} \cos \left(\omega_{n} \tau\right)\right. \\
& \left.+Q_{\mu, n} \sin \left(\omega_{n} \tau\right)\right) .
\end{aligned}
$$

Hence, by approximating the "action' functional $S$, one gets certain approximations $G_{N}(x, y)$ to the exact Green's function. These approximations converge to the exact Green's function [1] in the limit $N \rightarrow \infty$,

$$
G_{c}(x, y)=\lim _{N \rightarrow \infty} G_{N}(x, y),
$$

as soon as $\xi_{\mu}^{N}$ converges to $\xi_{\mu}$.

If all the $P_{n}$ 's are dropped in expressions (2) and (7), one gets a semiclassical approximation for which the path integration goes over the solutions to the classical "map,' Eqs. (4) and (3) with $\xi_{\mu}=0$ :

$$
m \frac{d x_{\mu}}{d \tau}-p_{\mu}-g A_{\mu}(x)=0
$$

where $\tau=2 \mathrm{~ms}$ is the proper time of the particle. Assuming that the particle is on its mass shell, one can also write the $\tau$ derivative of the last equation in terms of the electromagnetic field tensor $F_{\mu \nu}$ as

$$
\frac{d^{2} x_{\mu}}{d^{2} \tau}-g F_{\mu \nu} \frac{d x_{\nu}}{d \tau}=0
$$

In general, the possibility of sequentially dropping the $P_{n}$ dependence in Eq. (4), that is to say, of introducing the quantum fluctuations gradually, allows one to make some close observations of the corresponding quantum dynamics. 
Another way to look at the possibility of approximating the Green's function by using a certain Fourier polynomial of $\xi_{\mu}(\tau)$ is to say that the approximating procedure induces an ordering among the configurational space paths. According to the amount of contribution the $\gamma$ 's make to the integral (2), one can introduce a partition of the total path set $\Gamma(M)$ as the following: a path $\gamma:\left\{X_{\mu}(\tau), 0 \leqslant \tau \leqslant T\right\}$ in the configurational space will belong to a set $\Gamma_{N} \subset \Gamma(M)$ if it satisfies the constraint

$$
\begin{aligned}
\frac{d X_{\mu}}{d \tau}+g A_{\mu}(X)= & \frac{p_{\mu}+p_{\mu}^{\prime}}{2}+\frac{1}{\sqrt{T}} \sum_{n=0}^{N}\left(P_{\mu, n} \cos \left(\omega_{n} \tau\right)\right. \\
& \left.+Q_{\mu, n} \sin \left(\omega_{n} \tau\right)\right)
\end{aligned}
$$

for some finite number $N$ of harmonics and arbitrary values of the coefficients $P_{n}$. Obviously, the set $\Gamma_{0}$, obtained by keeping only $P_{0}=p_{\mu}+p_{\mu}^{\prime} / 2$, while $P_{n}=0$ for $n>0$, corresponds to the totality of the classical trajectories of the system. The corresponding eikonal approximation is just the semiclassical Green's function. The next approximation set will correspond to $P_{n}=0, n>1$, in which case, in order to evaluate the $G_{1}(x, y)$ one should consider all the paths that satisfy

$$
\begin{aligned}
\frac{d X_{\mu}}{d \tau}+g A_{\mu}(X)= & \frac{p_{\mu}+p_{\mu}^{\prime}}{2}+\frac{1}{\sqrt{T}}\left(P_{\mu, 1} \cos \left(\omega_{1} \tau\right)\right. \\
& \left.+Q_{\mu, 1} \sin \left(\omega_{1} \tau\right)\right),
\end{aligned}
$$

for all possible values $-\infty \leqslant P_{1}, Q_{1} \leqslant \infty$. The next approximation $G_{2}$ requires two harmonics $\left(P_{1}, Q_{1}, P_{2}, Q_{2}\right) \neq 0$, with the previous case corresponding to the particular parameter value subset $\left(P_{2}=Q_{2}=0\right)$ and so on. Obviously $\Gamma_{0} \subset \Gamma_{1} \subset \Gamma_{2} \cdots \subset \Gamma_{N} \subset \cdots \subset \Gamma_{\epsilon} \equiv \Gamma(M)$.

It is important to mention that from the point of view of the dynamics of the effective system (7a), adding the terms

$$
\begin{aligned}
\xi_{\mu} & =\frac{1}{2 \sqrt{T}} \sum_{n=-N}^{N} P_{\mu, n} e^{i \omega_{n} \tau} \\
& \equiv \frac{1}{\sqrt{T}} \sum_{n=0}^{N}\left(P_{\mu, n} \cos \left(\omega_{n} \tau\right)+Q_{\mu, n} \sin \left(\omega_{n} \tau\right)\right)
\end{aligned}
$$

to the right-hand sides of the classical dynamical equations certainly changes their dynamical behavior. For example, it can destroy the integrability of the classical equations,

$$
\frac{d X_{\mu}}{d \tau}+g A_{\mu}(X)=\frac{p_{\mu}+p_{\mu}^{\prime}}{2}
$$

and in general, the geometry of the trajectories of Eq. (10) can be completely different from that of the solutions to Eq. (9). In the first approximation, along with classical solutions to Eq. (9), $\left(P_{n}=0\right)$ one also considers more complicated paths that correspond to the solutions to Eq. (9) for $\left(P_{n}, Q_{n}\right) \neq 0, n>1$. The more harmonics that are kept in Eq. (9), the more geometrically complicated paths can occur among the solutions to Eq. (9), and in the limit $N \rightarrow \infty$ all the paths $\gamma \in \Gamma_{\infty} \equiv \Gamma(M)$ contribute. What is important, how- ever, is that one naturally obtains an ordering of the paths according to their complexity.

Remarkably, the function $\xi_{\mu}$ was introduced in such a way that it does not contain any particular characteristics of the underlying classical system; so it is a universal system, an independent way to introduce quantum fluctuations. Physically one can visualize the effect of the "quasipotential' $\xi_{\mu}$ ( $P_{n}$ 's and $Q_{n}$ 's) by imagining that the particle is moving in a field of many infinitely uniform waves (7a), added to the background potential. Since one can consider a simplified dynamics by leaving only a finite number of $P_{n}$ 's and $Q_{n}$ 's in Eq. (4), it is possible to introduce the quantum fluctuations "gradually", into a system.

\section{APPLICATIONS: THE TRACE FORMULAS}

One of the most important features of a quantum system is the distribution of the energy levels. The distribution of these levels is expected to be different depending on whether the underlying classical system is chaotic or integrable. To work with the energy level distribution, it is convenient to introduce the quantity

$$
\begin{aligned}
G(E) & =\operatorname{Tr} \frac{1}{\hat{H}-E}=\operatorname{Tr} G(x, y \mid E)=\int G(x, x \mid E) d x \\
& =\sum_{n} \frac{1}{E_{n}-E} .
\end{aligned}
$$

In most of the cases, especially in the nonintegrable ones, the explicit dependence of the energy levels on quantum numbers is unknown. In such cases the latter expression provides an (essentially unique) method of finding the eigenvalues of $\hat{H}$ as the poles of the function $G(E)$, if one manages to evaluate it in some other way.

There exist several methods of finding the trace $G(E)$ in the $\hbar \rightarrow 0$ limit [6]. The most acknowledged among them is, perhaps, Gutzwiller's trace formula, which states that in the quasiclassical limit, $G(E)$ is given by

$$
G_{q c l}(E)=\sum_{\text {per.orbits }} \frac{1}{\sqrt{\operatorname{det}\left(1-\mathbf{M}_{p}\right)}} e^{i S_{c l}+i \nu}
$$

where $\mathbf{M}$ is the stability matrix of the dynamical flow of the classical equations $\left(\delta S_{c l}=0\right)$ and $\nu$ is the Maslov index [5].

There exists an alternative approach discussed by Cvitanovic and others [7], who proposed to consider the trace of the classical transfer operator

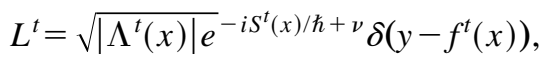

where $y=f^{t}(x)$ is the dynamical flow of the system corresponding to the action $S^{t}(x)$ and $\Lambda^{t}(x)$ is the expanding eigenvalue of the Jacobian transverse to the flow. Due to the $\sigma$ functional, the trace of the operator $L^{t}$ contains

$$
1 / \operatorname{det}\left(1-\mathbf{M}_{p}\right)
$$

instead of $1 / \sqrt{\operatorname{det}\left(1-\mathbf{M}_{p}\right)}$ as in Gutzwiller's formula [7], which affects the convergence rate of the sum over the classical orbits. An expression containing (13) was introduced in 
[7] as a regularization fo the sum (12), where the power of the pre-exponential factor was changed to improve the convergence properties of (12) and the analyticity of the trace $G(E)$

Conveniently, the possibility of dropping certain coefficients $P_{n}, Q_{n}$ in the formulas (2), (7a) or (7b) while keeping the $\delta$-functional constraint, allows one to derive the trace formulas for the successive approximations $G_{N}(x, y \mid E)$,

$$
G_{N}(E)=\operatorname{Tr} G_{N}(x, y \mid E)
$$

to approximate the exact trace $\operatorname{Tr} G(x, y \mid E)$. (7b):

For the 0th approximation $P_{n}=Q_{n}=0$, one gets from Eq.

$$
\begin{aligned}
\operatorname{Tr} G_{0}= & i \operatorname{Tr} \int_{0}^{\infty} d \tau e^{-i \tau m} \oint D X_{\mu} e^{i \oint\left(m \dot{X}_{\mu} / 2+g A_{\mu}(X)\right) d X_{\mu}} \\
& \times \delta\left(X_{\mu}-f_{\mu}^{\tau}(X, A, \xi=0)\right) \\
& \times\left\{m-i \gamma_{\mu}\left(g A_{\mu}(X)+p_{\mu}\right)\right\}\left(e^{-g \oint \sigma_{\mu \nu} F_{\nu \mu}(X)}\right)_{+} .
\end{aligned}
$$

Here all the paths satisfy $X_{\mu}(0)=X_{\mu}(T)$. Since $\sigma_{\mu \nu}$ is quadratic in Dirac matrices $\gamma_{\mu}$, the expansion of $e^{-g\} \sigma_{\mu \nu} F_{\nu \mu}}$ in the previous expression, combined with the factor $\gamma_{\mu}\left(g A_{\mu}(X)+p_{\mu}^{\prime}\right)$, produces only odd powers of $\gamma_{\mu}$, which have zero trace. Due to the $\delta$ functional the path integration goes over the solutions to the equations equivalent to the classical equations of motion:

$$
\frac{d x_{\mu}}{d s}+p_{\mu}-g A_{\mu}(x)=0 .
$$

Integrating over the classical periodic orbits $\gamma \in \Gamma_{0}$, one gets

$$
\begin{aligned}
& \operatorname{Tr} G_{0}= i \operatorname{Tr} \int_{0}^{\infty} d \tau e^{-i \tau m} \oint D X_{\mu} e^{i \phi\left(m \dot{X}_{\mu} / 2+g A_{\mu}\right) d X_{\mu}} \\
& \times \delta\left(X_{\mu}-f_{\mu}^{\tau}(\xi=0)\right)\left(e^{\left.-g \oint \sigma_{\mu \nu} F_{\nu \mu}\right)_{+}}\right. \\
&= \sum_{\text {clos.P.O. }} \frac{1}{\operatorname{det}\left(1-\mathbf{M}_{p}^{(0)}\right)} e^{-i S-i \nu} \\
& \equiv \sum_{P . O \in \Gamma_{0}} \frac{1}{\operatorname{det}\left(1-\mathbf{M}_{p}^{(0)}\right)} e^{-i S-i \nu}
\end{aligned}
$$

where

$$
S=\oint_{\gamma}\left(\frac{m}{2}+\frac{m \dot{X}_{\mu}^{2}}{2}+g A_{\mu}(X) \dot{X}_{\mu}+g \sigma_{\mu \nu} F_{\nu \mu}(X)\right) d \tau
$$

is the action, and $\mathbf{M}_{\mu \nu}^{(0)}$ is the stability matrix given by the flux of the equations (14), evaluated along each periodic orbit $\gamma \in \Gamma_{0}$. The pre-exponential determinant appeared naturally after integration over the path $D X_{\mu}$.

This result formally resembles the trace formula of Cvitanović and others [7]. The next approximation for $G_{1}(p)$ will produce in the same way

$$
\operatorname{Tr} G_{c}^{(1)}=\sum_{P . O \in \Gamma_{1}} \frac{1}{\operatorname{det}\left(1-\mathbf{M}_{p}^{(1)}\right)} e^{-i S-i \nu_{1}},
$$

and in general, one gets

$$
\operatorname{Tr} G_{c}^{(N)}=\sum_{P . O \in \Gamma_{N}} \frac{1}{\operatorname{det}\left(1-\mathbf{M}_{p}^{(N)}\right)} e^{-i S-i \nu_{N}},
$$

where $\mathbf{M}_{p}^{(N)}$ is the stability matrix evaluated along the periodic orbits of the system (9).

\section{SUMMARY}

The representations (7a) and (7b) are quite remarkable in many respects. First, due to the "effective dynamical system constraint" (3), the path integration always goes along the solutions to a certain dynamical system. Although for the exact representation this constraint is merely formal, since it does not actually narrow the set of paths used to evaluate the path integrals (7a) and (7b), it is very essential for the approximations $G_{N}$. There it becomes quite tangible, because the set of paths satisfying the condition (9) for $N<\infty$ is a small subset of the total set $\Gamma$ of the configurational space paths. Since $G_{N}$ 's converge rapidly to the exact Green's function, this observation allows one to group the paths in subsets $\Gamma_{N}$ according to the significance of the contribution they make to the integrals (7a) and (7b). As can be seen from the representation (7a), the approximations $G_{N}$ are nonperturbative and, therefore, they can be quite valuable tools for dealing with specific physical problems, such as those of quantum chaos. The usual tools for such analysis, the trace formulas, can be derived easily for every approximation $G_{N}$. It should be emphasized that the semiclassical trace formula (15) as well as its $\hbar \neq 0$ generalizations (16) and (17) were obtained using strictly nonperturbative techniques. Although there exist certain WKB-type approximations to the Green's function for $\hbar \neq 0$ case [8], they all come in the form of perturbation series in the powers of $\hbar$. A common shortcoming of such perturbative approximations are poor convergence properties and the lack of a clear geometrical interpretation of the expansion terms-except for the zeroth order term, the pure semiclassical case. Also, as was pointed out in [1], from a physical point of view such methods might not be adequate to describe certain features of quantum systems. Perturbative approaches, based on expansions of the functional integral near the saddle point, can destroy the evidence of certain dynamical effects, such as (quantum) chaos. On the other hand, a completely different approximation principle obtained in publications [1] allows one to get a sequence of approximations to the Green's function, without losing a geometrical perspective on the problem.

\section{APPENDIX A: A DERIVATION OF THE REPRESENTATION $(I)$}

Here we present the derivation of the expression (7a), following the notations of [1] and [3].

One writes the expression for Green's function in the momentum representation: 


$$
\begin{aligned}
& G_{c}\left(p, p^{\prime} \mid A\right)=i \int_{0}^{\infty} d s \int d^{4} z e^{-i s m^{2}-i q z+\left(i s q^{2} / 4\right)} \Pi_{n} \\
& \times \iint \frac{d^{4} P_{n} d^{4} Q_{n}}{(2 \pi)^{4}} e^{(i / 2)\left(P_{n}^{2}+Q_{n}^{2}\right)} \\
& \times \int D \Omega_{\mu} e^{-i \int_{0}^{s}(p-\Omega)^{2} d s^{\prime}} \\
& \times e^{-g \int_{0}^{s} d s^{\prime}\left(\partial / \partial z_{\mu}\right) A_{\mu}\left(\zeta(s)-2 \int_{0}^{s} d s^{\prime} \Omega\right)} \\
& \times\left\{m-i \gamma_{\mu}\left[p_{\mu}-\Omega_{\mu}\right]\right\} \\
& \times\left(e^{-g \int \sigma F\left(\zeta(s)-2 \int_{0}^{s} d s^{\prime} \Omega\right)}\right)_{+},
\end{aligned}
$$

where $\Omega_{\mu}(s)$ satisfies the constraint (4)

$$
\phi_{\mu}(\Omega, A) \equiv \Omega_{\mu}\left(s^{\prime}\right)-g A_{\mu}\left(\zeta\left(s^{\prime}\right)-2 \int_{0}^{s^{\prime}} d s^{\prime \prime} \Omega\left(s^{\prime \prime}\right)\right)=0
$$

The $\zeta_{\mu}\left(s^{\prime}\right)$ and $R_{\mu}\left(s^{\prime}\right)$ are defined as

$$
\begin{gathered}
\zeta_{\mu}\left(s^{\prime}\right)=z_{\mu}+s^{\prime}\left(p_{\mu}+p_{\mu}^{\prime}\right)-2 R_{\mu}\left(s^{\prime}\right), \quad q_{\mu}=p_{\mu}-p_{\mu}^{\prime}, \\
\mu=0,1,2,3 \\
R_{\mu}\left(s^{\prime}\right)=\frac{1}{2 \pi} \sum_{n} \frac{\sqrt{s}}{(n+1 / 2)}\left[P_{\mu, n} \cos \left(\frac{2 \pi s^{\prime}}{s}(n+1 / 2)\right)\right. \\
\left.+Q_{\mu, n} \sin \left(\frac{2 \pi s^{\prime}}{s}(n+1 / 2)\right)\right]
\end{gathered}
$$

It will be more convenient, however, to work with the derivative of $R_{\mu}\left(s^{\prime}\right)$,

$$
\begin{aligned}
\frac{d R_{\mu}\left(s^{\prime}\right)}{d s^{\prime}} & \equiv \xi_{\mu}\left(s^{\prime}\right) \\
& =\frac{1}{\pi \sqrt{s}} \sum_{n}\left(Q_{\mu, n} \cos \left(\omega_{n} s^{\prime}\right)-P_{\mu, n} \sin \left(\omega_{n} s^{\prime}\right)\right) .
\end{aligned}
$$

The exponent

$$
e^{-g \int_{0}^{s} d s^{\prime}\left(\partial / \partial z_{\mu}\right) A_{\mu}\left(\zeta(s)-2 \|_{0}^{s} d s^{\prime} \Omega\left(s^{\prime}\right)\right)}
$$

can be written symbolically as

$$
\begin{aligned}
e^{-g \int_{0}^{s} d s^{\prime}\left(\partial / \partial z_{\mu}\right) A_{\mu}\left(\zeta(s)-2 \int_{0}^{s} d s^{\prime} \Omega\left(s^{\prime}\right)\right)} & =e^{i t r \ln \left(\delta \phi_{\mu}\left(s^{\prime}\right) / \delta x_{\nu}\left(s^{\prime \prime}\right)\right)} \\
& =\operatorname{det}\left(\frac{\delta \phi_{\mu}\left(s^{\prime}\right)}{\delta x_{\nu}\left(s^{\prime \prime}\right)}\right)
\end{aligned}
$$

One has

$$
\begin{aligned}
G_{c}= & i \int_{0}^{\infty} d s \int d^{4} z e^{-i s m^{2}-i q z+\left(i s q^{2} / 4\right)} \Pi_{n} \\
& \times \iint \frac{d^{4} P_{n} d^{4} Q_{n}}{(2 \pi)^{4}} e^{i / 2\left(P_{n}^{2}+Q_{n}^{2}\right)} e^{-i \int_{0}^{s}(p-\Omega)^{2} d s^{\prime}} \\
& \times \operatorname{det}\left(\frac{\delta \phi_{\mu}\left(s^{\prime}\right)}{\delta x_{\nu}\left(s^{\prime \prime}\right)}\right)\{m-i \gamma[p-\Omega(s)]\} \\
& \times\left(e^{-g \int \sigma F\left(\zeta(s)-2 \int_{0}^{s} d s^{\prime} \Omega\left(s^{\prime}\right)\right)}\right)_{+} .
\end{aligned}
$$

Note, that one can obtain the determinant $\operatorname{det}\left(\delta \phi_{\mu}\left(s^{\prime}\right) / \delta x_{\nu}\left(s^{\prime \prime}\right)\right)$ by inserting formally the $\delta$ functional,

$$
\delta\left(\int_{0}^{s} \Omega_{\mu}\left(s^{\prime}\right)-g \int_{0}^{s} A_{\mu}\left(\zeta\left(s^{\prime}\right)-2 \int_{0}^{s^{\prime}} d s^{\prime \prime} \Omega\left(s^{\prime \prime}\right)\right)\right),
$$

and integrating over the $\Omega_{\mu}\left(s^{\prime}\right)$ :

$$
\begin{aligned}
G_{c}= & i \int_{0}^{\infty} d s \int d^{4} z \int D \Omega_{\mu} e^{-i s m^{2}-i q z+\left(i s q^{2} / 4\right)} \Pi_{n} \\
& \times \iint \frac{d^{4} P_{n} d^{4} Q_{n}}{(2 \pi)^{4}} e^{(i / 2)\left(P_{n}^{2}+Q_{n}^{2}\right)} e^{-i \int_{0}^{s}(p-\Omega)^{2} d s^{\prime}} \\
& \times\{m-i \gamma[p-\Omega]\} \delta\left(\int_{0}^{s} \Omega_{\mu}\left(s^{\prime}\right)-g \int_{0}^{s} A_{\mu}\right. \\
& \left.\times\left(\zeta\left(s^{\prime}\right)-2 \int_{0}^{s^{\prime}} d s^{\prime \prime} \Omega\right)\right)\left(e^{-g \int \sigma F\left(\zeta(s)-2 \int_{0}^{s} d s^{\prime} \Omega\right)}\right)_{+} .
\end{aligned}
$$

Now it is natural to call the whole argument of $A_{\mu}$ a new of variable $X_{\mu}$ :

$$
X_{\mu}\left(s^{\prime}\right)=z_{\mu}-s^{\prime} q_{\mu}-2 R_{\mu}\left(s^{\prime}\right)-2 \int_{0}^{s^{\prime}}\left(\Omega_{\mu}\left(s^{\prime \prime}\right)-p_{\mu}\right) d s^{\prime \prime}
$$

It follows from the previous line that

$$
\frac{1}{2} \frac{d X_{\mu}\left(s^{\prime}\right)}{d s^{\prime}}+\frac{q_{\mu}}{2}+\frac{d R_{\mu}\left(s^{\prime}\right)}{d s^{\prime}}=-\left(\Omega_{\mu}\left(s^{\prime}\right)-p_{\mu}\right) .
$$

Squaring both sides of Eq. (A4), one gets

$$
\begin{gathered}
\frac{1}{4} \dot{X}_{\mu}^{2}\left(s^{\prime}\right)+\frac{q_{\mu}^{2}}{4}+\dot{R}_{\mu}^{2}\left(s^{\prime}\right)+\frac{1}{2} q_{\mu} \dot{X}_{\mu}\left(s^{\prime}\right)+q_{\mu} \dot{R}_{\mu}\left(s^{\prime}\right) \\
+\dot{R}_{\mu}\left(s^{\prime}\right) \dot{X}_{\mu}\left(s^{\prime}\right)=\left(\Omega_{\mu}\left(s^{\prime}\right)-p_{\mu}\right)^{2},
\end{gathered}
$$

which yields, after integrating over $d s^{\prime}$ from 0 to $s$ [the same interval $0<s^{\prime}<s$ over which the expansion of $R_{\mu}(s)$ is defined], 


$$
\begin{aligned}
0= & \frac{1}{4} \int_{0}^{s} \dot{X}_{\mu}^{2} d s^{\prime}+\frac{q_{\mu}}{2}\left(\Delta X_{\mu}(s)\right)+q_{\mu}\left(\Delta R_{\mu}(s)\right)+\int_{0}^{s} \dot{R}_{\mu} \dot{X}_{\mu} d s \\
& -\left(\int_{0}^{s}\left(\Omega_{\mu}\left(s^{\prime}\right)-p_{\mu}\right)^{2} d s^{\prime}-\frac{q_{\mu}^{2} s}{4}-\int_{0}^{s} \dot{R}_{\mu}^{2} d s\right), \quad \text { (A5) }
\end{aligned}
$$

where $\quad \Delta X_{\mu}(s)=X_{\mu}(s)-X_{\mu}(0) \quad$ and $\quad \Delta R_{\mu}(s)=R_{\mu}(s)$ $-R_{\mu}(0)$. has

From the definition of the (functional) variable $R_{\mu}$, one

$$
\int_{0}^{s} \dot{R}_{\mu}^{2} d s=\frac{1}{2}\left(P_{n}^{2}+Q_{n}^{2}\right)
$$

since the $P_{n} \cos \left[(2 n+1) \pi s^{\prime} / s\right]$ and $Q_{n} \sin \left[(2 n+1) \pi s^{\prime} / s\right]$ terms are orthogonal on the interval $0 \leqslant s^{\prime} \leqslant s$ for the different $n$ 's.

Now one can see that all the three terms in parentheses in the second line of the expression (A4) appear in the arguments of the exponents in the expression (A1), which allows one to rewrite the Green's function as

$$
\begin{aligned}
G_{c}= & i \int_{0}^{\infty} d s \int d^{4} z e^{-i s m^{2}-i q z} \int D \Omega_{\mu} \\
& \times \int d R_{\mu} e^{-(i / 4) \int \dot{X}_{\mu}^{2} d s^{\prime}} e^{-i(q / 2) \Delta X-i q \Delta R-i \int \dot{X}_{\mu} \dot{R}_{\mu} d s^{\prime}} \\
& \times \delta\left(X_{\mu}-f_{\mu}^{s}(X, A, R)\right)\left\{m-i \gamma_{\mu}\left(\frac{d X_{\mu}}{2 d s}+\frac{q_{\mu}}{2}+\frac{d R_{\mu}}{d s}\right)\right\} \\
& \times\left(e^{-g \int \sigma F(X)}\right)_{+} .
\end{aligned}
$$

One can collect the two integrals in the arguments of the exponents in the previous expression into a single phase:

$$
\begin{aligned}
G_{c}= & i \int_{0}^{\infty} d s \int d^{4} z e^{-i m^{2} s} \int D \Omega_{\mu} \\
& \times \int d R_{\mu} e^{-i \int_{\gamma}\left(\dot{X}_{\mu} / 4+\dot{R}_{\mu}\right) d X}{ }_{\mu} e^{-i(q / 2) \Delta X-i q \Delta R-i q z} \\
& \times \delta\left(X_{\mu}-f_{\mu}^{s}(X, A, R)\right)\left\{m-i \gamma_{\mu}\left(\frac{d X_{\mu}}{2 d s}+\frac{q_{\mu}}{2}+\frac{d R_{\mu}}{d s}\right)\right\} \\
& \times\left(e^{-g \int \sigma F(X)}\right)_{+},
\end{aligned}
$$

where the integration in $\int_{\gamma}\left(\dot{X}_{\mu} / 4+\dot{R}_{\mu}\right) d X_{\mu}$ goes over a path connecting the $X_{\mu}(0)$ and $X_{\mu}(s)$ - the initial and the final points of the evolution. To clarify the geometrical meaning of the phase of the exponent $e^{-i(q / 2) \Delta X-i q \Delta R-i q z}$, one considers the explicit definitions of the functions $X_{\mu}$ and $R_{\mu}$ :

$$
\begin{gathered}
R_{\mu}(0)=\sum_{n} \frac{\sqrt{s}}{\pi(2 n+1)} P_{\mu, n}, \\
R_{\mu}(s)=\sum_{n} \frac{\sqrt{s}}{\pi(2 n+1)}(-1)^{(2 n+1)} P_{\mu, n} \\
=-\sum_{n} \frac{\sqrt{s}}{\pi(2 n+1)} P_{\mu, n}=-R_{\mu}(0) .
\end{gathered}
$$

So

$$
\Delta R_{\mu}(s)=R_{\mu}(s)-R_{\mu}(0)=-2 R_{\mu}(0) .
$$

On the other hand,

$$
X_{\mu}(0)=z_{\mu}-2 R_{\mu}(0)
$$

$$
X_{\mu}(s)=z_{\mu}-s q_{\mu}+2 R_{\mu}(0)-2 \int_{0}^{s}\left(\Omega_{\mu}\left(s^{\prime}\right)-p_{\mu}\right) d s^{\prime},
$$

so

$$
\begin{aligned}
\Delta X_{\mu}= & X_{\mu}(s)-X_{\mu}(0)=-s q_{\mu}+4 R_{\mu}(0) \\
& -2 \int_{0}^{s}\left(\Omega_{\mu}\left(s^{\prime}\right)-p_{\mu}\right) d s^{\prime},
\end{aligned}
$$

and, therefore,

$$
-i \frac{q_{\mu}}{2} \Delta X_{\mu}-i q_{\mu} \Delta R_{\mu}=i \frac{q_{\mu}^{2} s}{2}+q_{\mu} \int_{0}^{s}\left(\Omega_{\mu}\left(s^{\prime}\right)-p_{\mu}\right) d s^{\prime} .
$$

On the other hand, again using $R_{\mu}(s)=-R_{\mu}(0)$, one can write

$$
X_{\mu}(s)=2 z_{\mu}-s q_{\mu}-X_{\mu}(0)-2 \int_{0}^{s}\left(\Omega_{\mu}\left(s^{\prime}\right)-p_{\mu}\right) d s^{\prime}
$$

and

$$
X_{\mu}(s)+X_{\mu}(0)=2 z_{\mu}-s q_{\mu}-2 \int_{0}^{s}\left(\Omega_{\mu}\left(s^{\prime}\right)-p_{\mu}\right) d s^{\prime},
$$

Therefore,

$$
\begin{aligned}
-q_{\mu} & {\left[\frac{X_{\mu}(s)+X_{\mu}(0)}{2}\right]+q_{\mu} z_{\mu} } \\
& =i \frac{q_{\mu}^{2} s}{2}+q_{\mu} \int_{0}^{s}\left(\Omega_{\mu}\left(s^{\prime}\right)-p_{\mu}\right) d s^{\prime} .
\end{aligned}
$$

Comparing the right-hand sides of Eqs. (A7) and (A8), one can see that

$$
-i \frac{q_{\mu}}{2} \Delta X_{\mu}-i q_{\mu} \Delta R_{\mu}=-q_{\mu}\left[\frac{X_{\mu}(s)+X_{\mu}(0)}{2}\right]+q_{\mu} z_{\mu} ;
$$

so finally,

$$
i \frac{q_{\mu}}{2} \Delta X_{\mu}+i q_{\mu} \Delta R_{\mu}+q_{\mu} z_{\mu}=q_{\mu}\left[\frac{X_{\mu}(s)+X_{\mu}(0)}{2}\right] .
$$

As one can see, the argument of the third exponent $q_{\mu} z_{\mu}$ appeared in the last expression. Thus, 


$$
\begin{aligned}
G_{c}= & i \int_{0}^{\infty} d s \int d^{4} z e^{-i m^{2} s} \int D \Omega_{\mu} \\
& \times \int d R_{\mu} e^{-i \int_{\gamma}\left(\dot{X}_{\mu} / 4+\dot{R}_{\mu}\right) d X_{\mu}} e^{i\left(q_{\mu} / 2\right)\left(X_{\mu}(s)+X_{\mu}(0)\right)} \\
& \times \delta\left(X_{\mu}-f_{\mu}^{s}(X, A, R)\right)\left\{m-i \gamma_{\mu}\left(\frac{d X_{\mu}}{2 d s}+\frac{q_{\mu}}{2}+\frac{d R_{\mu}}{d s}\right)\right\} \\
& \times\left(e^{-g \int \sigma F(X)}\right)_{+} \cdot
\end{aligned}
$$

One can also simplify the measure of integration in the last expression in terms of the variables $X_{\mu}$ and $R_{\mu}$. As it is easy to see, the variable $X_{\mu}$ is a combination of two functionally independent parts:

$$
\begin{aligned}
X_{\mu}(s) & \equiv\left(z_{\mu}-s q_{\mu}-2 \int_{0}^{s}\left(\Omega_{\mu}\left(s^{\prime}\right)-p_{\mu}\right) d s^{\prime}\right)-\left(2 R_{\mu}\right) \\
& \equiv Z_{\mu}-2 R_{\mu},
\end{aligned}
$$

where

$$
\frac{\delta Z_{\mu}(s)}{\delta R_{\nu}\left(s^{\prime}\right)}=0, \quad \text { with } \quad Z_{\mu}(0)=z_{\mu}, \quad \text { and } R_{\mu}(0)=\sum_{n} P_{n}
$$

The total set of the integration variables in Eq. (A1) consists of $\left(d^{4} z_{\mu}, D \Omega_{\mu}, D R_{\mu}\right)$. The integration over $D \Omega$ and $d^{4} z_{\mu}$ in formula (A1) can be treated as an integration over the functional variable $Z_{\mu}$ :

$$
d^{4} z_{\mu} D \Omega_{\mu}=D Z_{\mu} .
$$

On the other hand, the functionals depend on the variables $X_{\mu}=Z_{\mu}-2 R_{\mu}$ and $R_{\mu}$. In order to integrate over these variables, one considers the following linear transformation at every moment $s^{\prime}$ :

$$
\left(\begin{array}{l}
X_{\mu} \\
R_{\mu}
\end{array}\right)=\left(\begin{array}{cc}
1 & -2 \\
0 & 1
\end{array}\right)\left(\begin{array}{l}
Z_{\mu} \\
R_{\mu}
\end{array}\right)
$$

Since this transformation is unimodular at every point $s^{\prime}$, one can write

$$
\begin{aligned}
G_{c}= & i \int_{0}^{\infty} d s e^{-i m s^{2}} \int D X_{\mu} \\
& \times \int d R_{\mu} e^{-i \int_{\gamma}\left(\dot{X}_{\mu} / 4+\dot{R}_{\mu}\right) d X_{\mu}} e^{i\left(q_{\mu} / 2\right)\left(X_{\mu}(s)+X_{\mu}(0)\right)} \\
& \times\left(e^{-g \int \sigma F(X)}\right)_{+} \delta\left(X_{\mu}-f_{\mu}^{s}(X, A, R)\right) \\
& \times\left\{m-i \gamma_{\mu}\left(\frac{d X_{\mu}}{2 d s}+\frac{q_{\mu}}{2}+\frac{d R_{\mu}}{d s}\right)\right\}
\end{aligned}
$$

In order to maintain the possibility of dropping certain $P_{n}$ and $Q_{n}$ dependencies in Eq. (A9) as in the original expression (A1), one has to make explicit the definition of the $R_{\mu}$ by inserting an additional $\delta$ functional,

$$
\begin{aligned}
& \delta\left(R_{\mu}-\sum_{n=1}^{N} \frac{1}{\sqrt{s}}\left[P_{\mu, n} \cos \left(\frac{(2 n+1) \pi s^{\prime}}{s}\right)\right.\right. \\
& \left.\left.+Q_{\mu, n} \sin \left(\frac{(2 n+1) \pi s^{\prime}}{s}\right)\right]\right),
\end{aligned}
$$

in the integral for $G_{c}$. This $\delta$ functional allows one to drop the coefficients $P_{n}$ and $Q_{n}, n>N$, in the expression for the Green's function while formally keeping the functional integration over $R_{\mu}$. If $N=\infty$, then Eq. (A10) is not necessary. In the case if some of the $P_{n}$ 's and $Q_{n}$ 's are omitted, then the constraint (A10) can be important. However, the determinants, which it will produce after $\dot{R}_{\mu}$ (or $\xi_{\mu}$ ) integration, are always equal to 1 , which means that the formula (A9), as well as its prototype, also allows one to drop certain $P_{n}$ and $Q_{n}$ dependencies.

\section{APPENDIX B: THE CASE OF THE RELATIVISTIC FREE PARTICLE}

To illustrate the "integration over the effective trajectories" method, it is appropriate to present here a simple example of how Eqs. (7a) and (7b) work in some simple cases. The simplest example would be the Green's function of a free particle, $A_{\mu}(x)=0$. For this case one has

$$
\begin{aligned}
G_{c}= & \frac{i}{2 m} \int_{0}^{\infty} d \tau \int D X_{\mu} \int D \xi_{\mu}\left\{m-i \gamma_{\mu} p_{\mu}\right\} \\
& \times e^{-(i / 2) \int_{0}^{T} m d \tau+(i m / 2)} \int_{\gamma} \dot{X}_{\mu}^{2} d \tau e^{-i\left(p_{\mu}^{\prime} X_{\mu}(s)-p_{\mu} X_{\mu}(0)\right)} .
\end{aligned}
$$

The functional $S$ in the exponent must be evaluated along the orbits of the system of equations,

$$
\frac{d X_{\mu}}{d \tau}=\frac{P_{\mu}}{2 m}-2 \xi_{\mu}
$$

or

$$
\begin{gathered}
\dot{X}_{\mu}=\frac{P_{\mu}}{2 m}-\sum_{n} \frac{2}{\sqrt{T}}\left[Q_{\mu, n} \cos \omega_{n} \tau-P_{\mu, n} \sin \omega_{n} \tau\right], \\
0 \leqslant \tau \leqslant T,
\end{gathered}
$$

where $\omega_{n}=\omega\left(n+\frac{1}{2}\right), \omega=2 \pi / T$, and $P_{\mu}=p_{\mu}+p_{\mu}^{\prime}$. The solutions to this system of equations are given by

$$
\begin{aligned}
X_{\mu}(\tau)= & z_{\mu}+\frac{\tau P_{\mu}}{2 m}-\frac{1}{\pi} \sum_{n} \frac{2 \sqrt{T}}{(2 n+1)} \\
& \times\left[P_{\mu, n} \cos \omega_{n} \tau+Q_{\mu, n} \sin \omega_{n} \tau\right],
\end{aligned}
$$

where $z_{\mu}$ is the integration constant, integration over which is assumed in $D X_{\mu}$. Hence,

$$
X_{\mu}(0)=z_{\mu}-\frac{1}{\pi} \sum_{n} \frac{2 \sqrt{T}}{(2 n+1)} P_{\mu, n}
$$




$$
X_{\mu}(T)=z_{\mu}+\frac{T P_{\mu}}{2 m}+\frac{1}{\pi} \sum_{n} \frac{2 \sqrt{T}}{(2 n+1)} P_{\mu, n},
$$

so

$$
\begin{aligned}
p_{\mu}^{\prime} X_{\mu}(T)-p_{\mu} X_{\mu}(0)= & z_{\mu} q_{\mu}+\frac{T P_{\mu} p_{\mu}^{\prime}}{2 m} \\
& +\frac{\sqrt{T} P_{\mu}}{\pi} \sum_{n} \frac{P_{\mu, n}}{(n+1 / 2)} .
\end{aligned}
$$

Using the relation $\omega_{n} T=(2 n+1) \pi$, one has for the phase

$$
\begin{aligned}
\frac{m}{2} \int_{0}^{T} \dot{X}_{\mu}^{2} d \tau-\frac{1}{2} \int_{0}^{T} m d \tau= & -\frac{i}{2} m T+m \sum_{n}\left(P_{\mu, n}^{2}+Q_{\mu, n}^{2}\right) \\
& +\frac{P_{\mu} \sqrt{T}}{\pi} \sum_{n} \frac{P_{\mu, n}}{n+\frac{1}{2}}+\frac{T P_{\mu}^{2}}{8 m}
\end{aligned}
$$

and the Green's function

$$
\begin{aligned}
G_{c}= & \frac{i}{2 m} \int_{0}^{\infty} d T \Pi_{n} \int d^{4} z \iint \frac{d^{4} P_{n} d^{4} Q_{n}}{(2 \pi)^{4}} e^{-(i / 2) m T+i m \Sigma_{n}\left(P_{\mu, n}^{2}+Q_{\mu, n}^{2}\right)+\left(\sqrt{T} P_{\mu} / \pi\right) \Sigma_{n} P_{\mu, n} /(n+1 / 2)+\mathrm{TP}_{\mu}^{2} / 8 m+i z_{\mu} q_{\mu}} \\
& \times\left\{m-i \gamma_{\mu} p_{\mu}\right\} e^{-i\left(T P_{\mu} p_{\mu}^{\prime} / 2 m+\left(\sqrt{T} P_{\mu} / \pi\right) \Sigma_{n} P_{\mu, n} /(n+1 / 2)\right)}
\end{aligned}
$$

The linear $P_{\mu, n}$ terms cancel out, and so

$$
\begin{aligned}
G_{c}= & \frac{i}{2 m} \int_{0}^{\infty} d T \Pi_{n} \int d^{4} z \iint \frac{d^{4} P_{n} d^{4} Q_{n}}{(2 \pi)^{4}} \\
& \times e^{-(i / 2) m T+i m} \sum_{n}\left(P_{\mu, n}^{2}+Q_{\mu, n}^{2}\right)+T P_{\mu}^{2} / 8 m+i z_{\mu} q_{\mu} \\
& \times\left\{m-i \gamma_{\mu} p_{\mu}\right\} e^{-i T P_{\mu} p_{\mu}^{\prime} / 2 m} .
\end{aligned}
$$

The Gaussian integral over the variables $P_{\mu, n}, Q_{\mu, n}$ is trivial, and the $z_{\mu}$ integration produces $\delta$ function $\delta\left(q_{\mu}\right)$, due to which one gets $q_{\mu}=0$, so $P_{\mu}=2 p_{\mu}$. Hence,

$$
\begin{aligned}
G_{c} & =\frac{i}{2 m} \int_{0}^{\infty} d T e^{-(i / 2) m T}\left\{m-i \gamma_{\mu} p_{\mu}\right\} e^{-i T p_{\mu}^{2} / m+i T p_{\mu}^{2} / 2 m} \\
& =i \int_{0}^{\infty} d \frac{T}{2 m}\left\{m-i \gamma_{\mu} p_{\mu}\right\} e^{-i\left(m^{2}+p_{\mu}^{2}\right) T / 2 m}=\frac{\left\{m-i \gamma_{\mu} p_{\mu}\right\}}{m+p_{\mu}^{2}} \\
& =\frac{1}{\gamma_{\mu} p_{\mu}+m}
\end{aligned}
$$

which is the Dirac equation Green's function.

\section{APPENDIX C: MOTION IN A CONSTANT MAGNETIC FIELD}

Another example provides a particle that moves in a constant electromagnetic field $F_{\mu \nu}=$ const, in which case the ordered exponent $\left(e^{g \int \sigma F}\right)_{+}$is just $e^{g \sigma_{\mu \nu} F_{\nu \mu} s}$. The vector potential $A_{\mu}$ is given by $A_{\mu}=-\frac{1}{2} F_{\mu \nu} x_{\nu}$. Consider for simplicity the case of the constant magnetic field in which case the vector potential in the symmetric gauge is

$$
A_{x}=-\frac{H}{2} y, \quad A_{y}=\frac{H}{2} x, \quad A_{z}=A_{0}=0 .
$$

The only nonzero component of $F_{\mu \nu}$ is $F_{31}=-F_{13}=H$, so $\sigma_{\mu \nu} F_{\nu \mu}=\sigma_{13} H$ :

$$
e^{g \sigma_{13} F_{31}}=e^{ \pm i g \sigma H} .
$$

It will be convenient to use the representation (7a), and evaluate the quantity

$$
\begin{aligned}
\widetilde{G}_{c}\left(x, x^{\prime}\right)= & i \int_{0}^{\infty} d s \int D X_{\mu} \\
& \times \int D \xi_{\mu} e^{-i m^{2} s} e^{-i \int_{\gamma}\left(\dot{X}_{\mu} / 4+A_{\mu}\right) d X_{\mu}} \\
& \times \delta\left(X_{\mu}-f_{\mu}^{s}(A)\right)\left(e^{-g \int \sigma F}\right)_{+},
\end{aligned}
$$

which produces the total Green's function through

$$
G_{c}\left(x, x^{\prime}\right)=\left(m-\gamma_{\mu} D_{\mu}\right) \widetilde{G}_{c}\left(x, x^{\prime}\right) .
$$

Using the $\delta$ constraint, it is easy to integrate formally over the $X_{\mu}(s)$. In this particular case it is possible to find the stability matrix $\mathbf{M}$ explicitly and evaluate the pre-exponential determinant $\operatorname{det}(1-\mathbf{M})$. For the equations

$$
\dot{X}_{\mu}=P_{\mu}-2 \xi_{\mu}-2 g A_{\mu}=P_{\mu}-2 \xi_{\mu}-g F_{\mu \nu} X_{\mu},
$$

the solution is

$$
\begin{aligned}
X_{\mu}\left(s^{\prime}\right)= & \left(e^{g F_{\mu \nu} s^{\prime}}\right) X_{\nu}(0)+P_{\nu} F_{\nu \mu}^{-1} \\
& -2 e^{g F_{\mu \nu} s^{\prime}} \int \xi_{\mu}\left(s^{\prime \prime}\right) e^{g F_{\mu \nu} s^{\prime \prime}} d s^{\prime \prime}
\end{aligned}
$$

and

$$
\operatorname{det}\left(\frac{\delta X_{\mu}\left(s^{\prime}\right)}{\delta X_{\mu}(0)}\right)=\operatorname{det}\left(1-e^{g F_{\mu \nu} s^{\prime}}\right)
$$

After the $X_{\mu}$ integration is carried through, one has to integrate over the solutions to the equations (3), which in this case are 


$$
\begin{gathered}
\frac{d X_{1}}{d s^{\prime}}=\frac{P_{1}}{2}-2 \xi_{1}+g H X_{2}, \\
\frac{d X_{2}}{d s^{\prime}}=\frac{P_{2}}{2}-2 \xi_{2}-g H X_{1}, \\
\frac{d X_{3}}{d s^{\prime}}=\frac{P_{3}}{2}-2 \xi_{3}, \\
\frac{d X_{0}}{d s^{\prime}}=\frac{P_{0}}{2}-2 \xi_{0} .
\end{gathered}
$$

The last two equations for $X_{3}$ and $X_{0}$ are separated and coincide with the free particle case. The first two equations yield

$$
\begin{gathered}
\ddot{X}_{1}+\omega_{0}^{2} X_{1}=\frac{\omega_{0} P_{2}}{2}-2 \dot{\xi}_{1}-2 \omega_{0} \xi_{2}, \\
\ddot{X}_{2}+\omega_{0}^{2} X_{2}=-\frac{\omega_{0} P_{1}}{2}-2 \dot{\xi}_{2}+2 \omega_{0} \xi_{1},
\end{gathered}
$$

where $\omega_{0}=g H$. By definition (7),

$$
\xi_{\mu}=\frac{1}{\sqrt{s}} \sum_{n}\left[Q_{\mu, n} \cos \omega_{n} s^{\prime}-P_{\mu, n} \sin \omega_{n} s^{\prime}\right],
$$

where $\omega_{n}=\omega(n+1 / 2), \omega=2 \pi / s$, and, therefore,

$$
\begin{aligned}
\ddot{X}_{1}+\omega_{0}^{2} X_{1}= & \frac{\omega_{0} P_{2}}{2}-\frac{2}{\sqrt{s}} \sum_{n}\left(\omega_{0} Q_{2, n}-\omega_{n} P_{1, n}\right) \cos \omega_{n} s^{\prime} \\
& +\frac{2}{\sqrt{s}} \sum_{n}\left(\omega_{0} P_{2, n}+\omega_{n} Q_{1, n}\right) \sin \omega_{n} s^{\prime}, \\
\ddot{X}_{2}+\omega_{0}^{2} X_{2}= & -\frac{\omega_{0} P_{1}}{2}+\frac{2}{\sqrt{s}} \sum_{n}\left(\omega_{0} Q_{1, n}+\omega_{n} P_{2, n}\right) \cos \omega_{n} s^{\prime} \\
& +\frac{2}{\sqrt{s}} \sum_{n}\left(\omega_{n} Q_{2, n}-\omega_{0} P_{1, n}\right) \sin \omega_{n} s^{\prime} .
\end{aligned}
$$

The solutions $X_{\mu}(s)$ to the equations above are given by

$$
\begin{aligned}
X_{1}= & V \sin \left(\omega_{0} s^{\prime}+\varphi\right)+\frac{P_{2}}{\omega_{0}} \\
& +\frac{2}{\sqrt{s}} \sum_{n}\left[\frac{\omega_{n} P_{1, n}-\omega_{0} Q_{2, n}}{\omega_{0}^{2}-\omega_{n}^{2}} \cos \omega_{n} s^{\prime}\right. \\
& \left.+\frac{\omega_{0} P_{2, n}+\omega_{n} Q_{1, n}}{\omega_{0}^{2}-\omega_{n}^{2}} \sin \omega_{n} s^{\prime}\right]
\end{aligned}
$$

$$
\begin{aligned}
X_{2}= & V \cos \left(\omega_{0} s^{\prime}+\varphi\right)-\frac{P_{1}}{\omega_{0}} \\
& +\frac{2}{\sqrt{s}} \sum_{n}\left[\frac{\omega_{0} Q_{1, n}+\omega_{n} P_{2, n}}{\omega_{0}^{2}-\omega_{n}^{2}} \cos \omega_{n} s^{\prime}\right. \\
& \left.+\frac{\omega_{n} Q_{2, n}-\omega_{0} P_{1, n}}{\omega_{0}^{2}-\omega_{n}^{2}} \sin \omega_{n} s^{\prime}\right]
\end{aligned}
$$

It is convenient to introduce new coefficients (the new variables of integration) $C_{1 n}, C_{2 n}, S_{1 n}, S_{2 n}$ as

$$
\begin{aligned}
X_{1}= & V \sin \left(\omega_{0} s^{\prime}+\varphi\right)+\frac{P_{2}}{\omega_{0}} \\
& +\frac{2}{\sqrt{s}} \sum_{n}\left(C_{1 n} \cos \omega_{n} s^{\prime}+S_{1 n} \sin \omega_{n} s^{\prime}\right), \\
X_{2}= & V \cos \left(\omega_{0} s^{\prime}+\varphi\right)-\frac{P_{1}}{\omega_{0}} \\
& +\frac{2}{\sqrt{s}} \sum_{n}\left(C_{2 n} \cos \omega_{n} s^{\prime}+S_{2 n} \sin \omega_{n} s^{\prime}\right),
\end{aligned}
$$

where

$$
\begin{aligned}
& Q_{1 n}=\omega_{0} C_{2, n}-\omega_{n} S_{1, n}, \quad P_{1 n}=-\omega_{0} S_{2, n}-\omega_{n} C_{1, n} \\
& Q_{2 n}=-\omega_{0} C_{1, n}-\omega_{n} S_{2, n}, \quad P_{2 n}=\omega_{0} S_{1, n}-\omega_{n} C_{2, n}
\end{aligned}
$$

The Jacobian of the transformation $\left(P_{1 n}, P_{2 n}, Q_{1 n}, Q_{2 n}\right)$ $\rightarrow\left(C_{1 n}, C_{2 n}, S_{1 n}, S_{2 n}\right)$ is $J=\left(\omega_{0}^{2}-\omega_{n}^{2}\right)^{2}$.

It is easy to observe that Eqs. (C1) and their solutions are equivalent to the ones that would correspond to the action,

$$
S=\int_{\gamma} \frac{\dot{X}_{\mu}^{2}}{4}+\frac{\omega_{0}^{2} X_{\mu}^{2}}{2} d t
$$

of a simple harmonic oscillator. The above expression and the one in the exponent of Eq. (C1) are equal up to the total derivative. Therefore, the dynamical properties of these systems are the same. The derivation of the function $\widetilde{G}_{c}$,

$$
\begin{aligned}
\widetilde{G}_{c}= & -\int_{0}^{\infty} \frac{d s}{(4 \pi s)^{2}} \frac{g H s}{\sin g H s} \\
& \times e^{-i\left(t-t^{\prime}\right)^{2} / 4 s+i\left(x_{3}^{\prime}-x_{3}\right)^{2} / 4 s} \\
& \times e^{i\left[(g H / 4)\left(\left(x_{1}-x_{1}^{\prime}\right)^{2}+\left(x_{2}-x_{2}^{\prime}\right)^{2}\right) \cot g H s+(g H / 2)\left(x_{1}+x_{1}^{\prime}\right)\left(x_{2}-x_{2}^{\prime}\right)\right],}
\end{aligned}
$$

follows the standard procedures [9], and produces the spectrum,

$$
E^{2}-m^{2} c^{2}-p_{z}^{2}=g H(2 n+1)-e H \sigma,
$$

of the Dirac equation energy spectrum of a charge $g$ moving in a constant magnetic field $H$. 
[1] H.M. Fried, Y. Gabelini, and B.H.J. McKellar, Phys. Rev. D 51, 7083 (1995); H.M Fried and Y. Gabelini, ibid. 51, 890 (1995); H.M. Fried, Y. Gabelini, and B.H.J. McKellar, Phys. Rev. Lett. 74, 4373 (1995).

[2] E.S. Fradkin, Nucl. Phys. 76, 588 (1966).

[3] H.M. Fried, Functional Methods and Eikonal Models (Edition Frontieres, Gif-sur-Yvette, France, 1990).

[4] C. Grosche and F. Steiner, Handbook of Feynman Path Integrals (Springer, Berlin, 1998); R.J. Rivers, Path Integral Methods in Quantum Field Theory (Cambridge University Press, Cambridge, 1987); Mark S. Swanson, Path Integrals and Quantum Processes (Academic Press, Boston, 1992).

[5] M. Gutzwiller, Chaos in Classical and Quantum Mechanics, (Springer-Verlag, Berlin, 1990); J.M. Robbins, Nonlinearity 4, 343 (1991).
[6] M.C. Gutzwiller, in The Origins of the Trace Formula, edited by editors, Vol. 485 of Lecture Notes in Physics (Springer, Berlin, 1997), p. 8; H. Primack and U. Smilansky, J. Phys. A 31, 6253 (1998); G. Vattay, Phys. Rev. Lett. 76, 1059 (1996).

[7] P. Cvitanović and B. Eckhard, J. Phys. A 24, L237 (1991); e-print chao-dyn/9303016 (1993); P. Cvitanović, G. Vattay, and A. Wirzba, e-print chao-dyn/9608012 (1996); P. Cvitanović and P.E. Rosenqvist, e-print chao-dyn/9304003 (1993).

[8] V.P. Maslov and M.V. Fedoriuk, Semi-Classical Approximations in Quantum Mechanics (D. Reidel, Dordrecht, 1981); A. Voros, in Semi-classical Approximations, Vol. XXIV of Annales de l'Institut Henri Poincaré-Section A (unpublished); G. Vattay, e-print chao-dyn/9406005.

[9] G.J. Papadopulos and J.T. Devreese, Phys. Rev. D 13, 2227 (1976). 